\title{
CAMBIAN LOS TIEMPOS, PERO ¿CAMBIAN LAS UNIVERSIDADES? LA EDUCACIÓN SUPERIOR ANTE LOS RETOS DE LA NUEVA SOCIEDAD
}

\author{
Joan Rué \\ IDES, UAB
}

\begin{abstract}
RESUMEN: Si una noción es recurrente, hoy, en el ámbito institucional universitario europeo, es la de cambio. Sin embargo, si se pudiera resumir de un modo gráfico una actitud ampliamente compartida por amplios sectores del profesorado, y aún de responsables institucionales, sería la que se enunciaría en los términos siguientes: “¿cambio?, ¿ qué cambio?".

En el presente artículo nos moveremos a lo largo del eje que definen ambas percepciones, la de que todo se está moviendo y aprisa y la que constata que nada se va a mover de forma relevante y que las cosas, en lo sustancial, van a ser lo mismo que ya conocemos. La posición que mantenemos en este texto es que, efectivamente, compartimos la perspectiva del cambio y de la naturaleza relevante de las fuerzas que lo impulsan, pero también consideramos que el mismo no va a darse necesariamente en un sentido profundo, y quizás quede como un maquillaje, a menos que se comprenda la naturaleza de las instituciones de educación superior, se analicen los rasgos centrales de su cultura y se diseñen y emprendan estrategias adecuadas, tanto desde el exterior como desde su propio interior, que permitan un desarrollo institucional congruente con las expectativas latentes en las demandas de cambio. En la parte final del texto se aportan algunas de las claves bajo las cuales se ha emprendido el camino del cambio en la UAB.
\end{abstract}

ABSTRACT: Change is the new mot d'ordre in the current international university context in Europe. But, beyond all the institutional statements there is still appropriate to consider this question: Change? What kind of change?.

In this paper we shall move along two main perceptions. The first one stresses that everything is moving forwards quickly and the second one underlines the fact that everything in universities tends to remain as it used to be, in its basic trends. In this paper we do stand, whit others, in sharing the changing perspective, specially because it is deeply related to the social forces that push forwards into that direction. Nevertheless, we are aware about the risk that promoted changes can be reduced into a simple institutional making up, unless we understand the nature of the High Education institutions, let's say its cultures, and, according to that, we also succeed in defining the best strategies that fit in such observed conditions and cultures. As a conclusion of the paper 
the reader will find some main keys that inspire the current process of change developed in the Barcelona Autonomous University (UAB).

PALABRAS CLAVE: Enseñanza superior, cambio social, ambiente social, universidad.

KEYWORDS: Higher education, social change, social enviroment, University.

\section{REFERENTES PARA COMPRENDER LA NATURALEZA PROFUNDA DEL CAMBIO APUNTADO EN LAS UNIVERSIDADES}

Parafraseando un conocido texto de Michael Fullan, podemos decir que, en la actualidad, se manifiestan fuerzas poderosas que apuntan hacia el cambio en la universidad, tanto en su estructura como en su función social. Sin embargo, la universidad que nosotros conocemos en nuestro país y en países vecinos, de naturaleza humboltiana, se ha ido desarrollando a lo largo de dos siglos sin apenas variaciones sustanciales, forjando una cultura institucional y unos hábitus ${ }^{1}$ profesionales de muy hondas raíces. Sin embargo, es a partir de los años ochenta cuando se empiezan a manifestar elementos significativos de cambio, cuyo diagnóstico ha cristalizado recientemente en una serie de documentos políticos ${ }^{2}$ que ya no sólo lo describen, sino que establecen una fecha límite para que las instituciones universitarias se transformen, adaptándose a los mismos.

¿Cuáles son estas fuerzas que orientan a la Universidad hacia el cambio? Examinemos algunas de las más relevantes.

1. La transformación cuantitativa radical de la demanda de formación superior, como consecuencia de la elevación progresiva del nivel de vida de la población que conlleva la ampliación de sus expectativas formativas, y de la necesidad de los ciudadanos de adaptarse a los requerimientos de un mercado de trabajo cambiante. Hoy, en Europa, se puede afirmar, generalizando un poco, que casi el $40 \%$ de la población juvenil entre 18 y 23 años se halla escolarizado en la Universidad. Un efecto de ello es que la naturaleza de la demanda de formación de esta universidad de masas es muy distinta a la proporcionada por la universidad de élites forjada a lo largo de los siglos. Dicha demanda ha llevado aparejada, además, la creación de numerosas universidades en los últimos años en nuestro país. Podemos dejar estos datos en una simple pregunta: ¿Cómo la cantidad no ha de afectar a la concepción de la propia universidad?

1. Tal como los define Bourdieu, P., (1997). Los hábitus “.son principios generadores de prácticas distintas y distintivas (lo que se hace y la forma de hacerlo), pero también son esquemas clasificatorios, principios de clasificación, principios de visión y de división, aficiones diferentes. Establecen diferencias entre lo es bueno y malo, entre los que está bien y está mal, entre lo distinguido y lo que es vulgar, etc., pero no son las mismas diferencias para unos y otros" (p:20). "El hábitus es esta especie de sentido práctico de lo que hay que hacer en una situación determinada" (p:40)"

2. El conjunto de esta documentación se conoce como el proceso Bolonia. 
2. La transformación política de los Estados y de muchas de sus atribuciones. En poco tiempo hemos podido constatar un doble proceso en Europa, el de su renovada ampliación, y el de la cesión por parte de los estados de importantes parcelas políticas no sólo funcionales, sino identitarias y estratégicas para las políticas económicas como la moneda, a organismos supranacionales como el Banco Europeo o la Comisión Europea. La eliminación de fronteras interiores y la libre circulación de personas y de profesionales hace que los modelos de formación y de certificación nacionales para las titulaciones sean ahora percibidos no como un derecho de los Estados, sino como verdaderos obstáculos al desarrollo del espacio científico y universitario en Europa.

3. La transformación de nuestras sociedades a lo largo de los últimos treinta años, al pasar de sociedades industriales más o menos avanzadas a sociedades del conocimiento (Castells, 1999). En las primeras, el poder descansa en la propiedad de los medios de producción. En las segundas, la clave del poder y del crecimiento es el valor añadido, la innovación y saber articularse en red. Estos aspectos, elementos determinantes del nuevo modelo productivo, van a tener una fuerte incidencia a medio plazo en el diseño de los modelos formativos, en sus finalidades y objetivos.

Si el trabajo es la fuente de la productividad, el poder creativo del trabajo y la eficiencia de la organización empresarial dependen en última instancia de la innovación. La innovación se halla en función de la mano de obra cualificada y de la existencia de organizaciones que sean generadoras de conocimientos. ... la innovación es la función principal (y) depende de la generación de conocimientos facilitada por el acceso abierto a la información (Castells, 2002:111)

En relación con lo anterior, cabe señalar el impacto enorme del crecimiento del conocimiento, juntamente con la aparición de la red a mediados de los ochenta y el enorme potencial que poseen las tecnologías de la información y de la comunicación como herramientas para almacenar, elaborar, tratar y comunicar el conocimiento.

4. Estrechamente relacionado con lo que se acaba de apuntar, encontramos también importantes cambios en la concepción misma de los períodos formativos. La estructura de la formación como un período cerrado pre-profesional y terminal se modifica. Salir de la universidad, especialmente en los países de Europa más avanzados y de modo creciente para muchas personas, es tan sólo un período de tiempo para volver a ella a formarse en Máster y Doctorado. El aprendizaje se visualiza ya como el telón de fondo para la vida productiva y la formación se contempla relacionada con el mercado de trabajo, lo que comporta flexibilidad y reconocimiento en valor de cambio para los distintos ciclos de la formación superior e incorpora al Doctorado como una etapa que integra y culmina la carrera formativa.

Una de las mayores implicaciones de la Declaración de Bolonia (1999) es la de potenciar estructuras académicas mucho más comprensibles y con menos barreras y diferencias a nivel europeo. Esto implica una mayor transparencia en el ámbito de las estructuras y de los programas educativos, lo que va a implicar una mayor competitividad entre universidades y centros debido al interés de mostrar unos programas cuya calidad les permita diferenciarse significativamente entre estas instituciones. El 
proceso iniciado desde la declaración de la Sorbona en 1998, y Bolonia el año siguiente, luego seguido en Praga, Berlín y Graz, ha terminado por caracterizar lo que los expertos (Haug, G., 2003) denominan "el triángulo de oro" de la reforma universitaria europea, el cual está configurado por un cambio de enfoque en las carreras, el cambio en la orientación de la enseñanza-aprendizaje, mediante los ECTS y la aparición de los sistemas de acreditación de la formación.

En este sentido, las nuevas líneas de acción que se proponen en los programas europeos (Haug, 2003) acentúan la creación de redes institucionales, la renovación curricular, el desarrollo de programas comunes y, de un modo especial, el desarrollo de una "etiqueta" de calidad para los programas acreditados, una etiqueta creíble y competitiva en todo el mundo.

En su conjunto, las grandes dinámicas apuntadas generan o van a generar un profundo cambio en el tipo de las demandas que se les hace a las instituciones de Educación Superior, así como al modo cómo deben ejecutarlas. En este sentido, y como concreción de aquellas, podemos señalar algunos de los rasgos emergentes o indicadores de la nueva demanda de formación superior:

- Los alumnos actúan como clientes en varios mercados o en varios segmentos del mercado de la formación.

- Surgen nuevas demandas desde los empleadores. Las empresas ahora piden a las instituciones universitarias trabajadores flexibles y conocimiento adaptable a las circunstancias variables de la producción. Esto se puede observar, por ejemplo, en los nuevos perfiles profesionales de muchas titulaciones que se han formulado a partir del proyectoPorta22@ en Barcelona. Dichos perfiles profesionales difieren significativamente en muchos aspectos de los perfiles bajo los que se orienta ahora mismo la formación proporcionada por las universidades radicadas en la misma área metropolitana.

- Los cambios económicos y las presiones del Mercado competitivo demandan mejores directrices que incluyan, según Gumport (2000), aspectos como un ajuste rápido de los programas, así como una mayor flexibilidad y eficiencia para una mayor satisfacción en los clientes ${ }^{3}$.

Sin embargo, el repertorio de cambios no se agota en la naturaleza de la demanda. Las universidades pasan a ser consideradas como una pieza más de la producción de conocimiento y del desarrollo. La investigación universitaria, tradicionalmente de carácter básico, pasa a tener las características de I+D, las instituciones se ven reguladas políticamente mediante programas de evaluación, mediante agencias externas, los cuales tienden a generar a la vez un sentido de responsabilización y de competitividad entre centros, así como a la necesidad de concertar compromisos de calidad con los gobiernos, "Contratos-Programa", a cambio de recursos.

3. Esta acepción del alumno, relativamente reciente en la literatura sobre universidades, constituye todo un indicador de la profundidad del cambio. Al margen del tipo de posición - de aceptación, de rechazo que suscite, indica que el alumno es un sujeto de derechos y que la financiación de las instituciones depende, en mayor o menor grado, de su elección y de permanencia, en definitiva de su satisfacción. 
Como síntesis de lo anterior, puede sernos de utilidad conocer el análisis de las insuficiencias del espacio europeo ante estas transformaciones según un reciente documento de la Comisión Europea, realizado desde la perspectiva que Estados y Universidades han definido en el proceso de convergencia europea (European Comisión 2003) :

- Se detecta un progreso insuficiente en estrategias para el aprendizaje a lo largo de la vida productiva.

- Se anuncia que no habrá una Europa del conocimiento sin una Europa de la Educación Superior

- La formación profesional se considera poco atractiva y de calidad insuficiente

- Poca movilidad en el campo de la formación y de la educación

- Se constata que la Unión queda atrás de sus principales competidores. Dicha constatación se focaliza en los aspectos siguientes: una insuficiente inversión en recursos humanos, en la falta de personas formadas en centros de educación superior, en el hecho de que la unión atrae menos personas de talento que sus competidores.

- El documento acentúa el hecho de que numerosos indicadores están todavía "en rojo", como la tasa de abandono escolar temprano en la universidad, cuyo objetivo para los próximos años es descender desde el 20 al 10\% . Otros indicadores son la tasa muy baja de mujeres en los campos científico y técnico, el hecho de que cerca del 20 de los jóvenes fracasan en la adquisición de competencias clave, en la constatación de que todavía pocos adultos participan de un aprendizaje continuado.

\section{LAS FUERZAS DEL CAMBIO Y LAS FUERZAS DE LA IDENTIDAD INSTITUCIONAL}

Si todo lo que acabamos de enunciar es tan potencialmente relevante para comprender la naturaleza del reto para el cambio al que las Universidades se enfrentan, como efectivamente lo es, ipor qué tantos agentes educativos perciben más la continuidad que la tensión para el cambio? ¿Por qué razón identifican las actuales turbulencias como un obstáculo que les impide realizar eficazmente su función, en vez de considerarlas signos de la crisis transformadora que se anuncia? ¿Qué rasgos poseen estas instituciones para ser aparentemente tan reactivas a lo anterior?

Diversas razones nos permetirían argumentarlo en un sentido explicativo. Entre ellas, señalaríamos el hecho de que no bastan las fuerzas emergentes por sí mismas para impulsar un cambio con sentido, si no existe un marco político explícito y resuelto que lo impulse, como empieza a ser evidente a partir de los documentos que van desarrollando los acuerdos de Bolonia . Otra razón es que estamos en los inicios de este proceso que se quiere ordenar mediante la presión política y que los principales efectos de la misma empezarán a ser visibles en los próximos años. Sin una dirección política a la altura de las circunstancias las fuerzas de cambio impulsarán dinámicas que tenderán a ser contempladas más como turbulencias que otra cosa.

Pero hay otras dos razones. La primera es que las denominadas demandas del mercado no siempre son claras ni se hallan orientadas en el sentido que la universidad las pueda recoger fácilmente tal como se está configurada, ni porque no está 
claro del todo que lo tenga que hacer, por lo menos atendiendo al enunciado de algunas formulaciones. Por ejemplo, ¿qué significa el término empleabilidad para titulaciones cuyo contenido es de orden más teórico que aplicado y donde los perfiles laborales requieren una serie de conocimientos que no se proporcionan de modo directo en la formación, como por ejemplo el dominio de la lengua inglesa? La universidad, tal como se halla organizada actualmente produce sus mejores efectos a medio y a largo plazo, en vez de a corto. Por todo ello, en la situación organizativa actual, el ciclo de adaptación a las demandas externas es necesariamente lento. Sin embargo, me detendré en una segunda razón, de naturaleza cultural, y que ejerce un importante efecto estabilizador, reactivo a los cambios.

La educación superior es una institución cuya tradicional razón de ser social ha sido preservar y desarrollar libremente la herencia educativa, así como cultivar el desarrollo de la civilización, la preservación del legado cultural y la formación de élites ilustradas. Dicha perspectiva, elaborada en el tiempo, es profunda y fortalece la visión de que la educación superior no puede adaptarse a ciertas demandas. Así, por ejemplo: ¿deben las Escuelas Superiores y las Facultades dirigirse de forma prioritaria a las necesidades económicas del mercado?, ¿Los estudiantes deben verse siempre como futuros empleados? ¿Pueden las universidades publicas sostener, a la vez, la transmisión de conocimiento, la producción y la creación básica como actividades compatibles? Una posición resultante de este enfoque vendría a decir que si se siguen las directrices marcadas por el mercado, centradas en las demandas económicas a corto plazo, no se podrán asumir las responsabilidades sociales comprometiendo de esta forma los intereses a largo plazo que la universidad tiene a nivel social.

Ello nos lleva a pensar que las reestructuraciones que se perfilan no pueden ser contempladas solo desde el punto de vista de los cambios de orden curricular y funcional, sino también como cambios institucionales cuya onda va a afectar en profundidad a los modelos organizativos desde los que se regulan los centros superiores. Ahora bien, dicho cambio no se logrará tan sólo por enunciarlo. Por ello, cobran una extraordinaria importancia los modelos que se apliquen para promover y acompañar a las instituciones de formación superior en el camino hacia el cambio institucional.

En cualquier caso, en esta confrontación entre estas dos fuerzas, las del cambio y las de la estabilización, hoy podemos contemplar cómo emergen problemas como los siguientes ${ }^{4}$ :

- Serias dudas acerca los estándares educativos, por parte de los formadores, pero también por parte de la sociedad y los empleadores, aunque cada una de las partes por razones distintas.

- Dudas acerca la eficiencia en el sistema de educación superior o la institución, entendida esta eficiencia desde la perspectiva del rendimiento de las inversiones públicas o de su impacto en el rendimiento económico de los alumnos, entendidos como recursos. 
- Dudas externas sobre la capacidad de innovación y dudas internas acerca de su necesidad.

- La necesidad de estimular sustancialmente el cambio cultural en las instituciones y la relativa ausencia de modelos y de cultura de innovación para hacerlo.

- La ausencia de recompensa y de retorno sobre los agentes y las instituciones en relación a lo que se hace, lo que afecta a la transparencia y a la responsabilización de las instituciones y de sus actividades.

- El traslado de ciertos costes del cambio, traducidos, a veces, en intensificación laboral, a los agentes universitarios singulares.

\section{CARACTERÍSTICAS DE LAS INSTITUCIONES DE EDUCACIÓN SUPERIOR}

Para comprender mejor la tensión entre las fuerzas del cambio y las de la estabilización, y poder comprender mejor cuáles serán las dinámicas resultantes de esta tensión, es interesante que nos detengamos en analizar cuáles son las principales características de las organizaciones de educación superior. Podríamos decir que normalmente, aunque en algunos países se den algunas diferencias con lo que decimos, el perfil de dichas instituciones puede ser reconocido a partir de los rasgos siguientes:

- Un importante grado de autonomía política institucional.

- Una distribución de los liderazgos institucionales diferentes a los de otras instituciones (Centros de investigación, Academias, Colegios profesionales, Empresas, etc.).

- La primera fuente de autoridad es el estatus profesional personal, lo que implica un papel débil de la dirección institucional.

- Un elevado grado de autonomía profesional.

- Roles profesionales altamente especializados.

- Grupos de académicos fuertemente vinculados a redes académicas cuyas dinámicas de acción o profesionalmente identitarias pueden ser autónomas de la institución.

- Un alto grado de diferenciación estructural a nivel de departamentos, por lo que cada departamento puede ser un mundo en si mismo.

- Un grado de equilibrio inestable o de conflicto más o menos latente entre las necesidades formativas (de Escuelas, Facultades, Unidades y titulaciones) y las de la investigación, y la asignación de recursos docentes, por parte de los Departamentos.

Un concepto de interés, aplicable a las instituciones educativas, es el de entenderlas como uniones sueltas (o loose coupling) tal como lo han formulado Glassman, en 1973 y Mach y Olsen en 1975, según Weick (1994:124-6). Con esta fórmula se quiere dar a entender que distintos sucesos mantienen una relación de correspondencia, a la vez que cada agente o cada unidad organizativa preserva su propia identidad y alguna evidencia física o lógica de su separación. De acuerdo con Weick, estas serían algunas de las propiedades funcionales de este concepto: 
- las uniones sueltas facilitan tanto la persistencia de elementos de organización ya superados, como que la organización se adaptará a ciertos cambios.

- facilitan nuevas adaptaciones situacionales de la organización.

- en un sistema en el cual los elementos preservan su identidad, su singularidad y separación, el sistema puede retener potencialmente una gran cantidad de mutaciones y de nuevas soluciones.

- cuando se produce una ruptura en una parte del sistema, éste no se resiente necesariamente en su integridad.

- en la medida que algunos de los elementos más importantes en las organizaciones educativas son profesores, clases, directivos, etc., en un sistema de uniones sueltas poseen un mayor margen de maniobrabilidad o de autonomía en el desempeño de sus respectivas competencias.

Weick (1994:123) apunta a que cuando se describen sistemas de uniones sueltas generalmente se refieren a aspectos como los siguientes:

- situaciones relajadas, cuando por ejemplo los recursos disponibles superan a las demandas que recibe una organización.

- ocasiones en las que cualquiera de entre muchos medios conduce al mismo final.

- una relativa falta de coordinación, o un nivel bajo en la misma.

- una relativa falta de regulaciones.

- situaciones de descentralización.

- nivel muy bajo de supervisión de las actividades.

A pesar de estos rasgos, dicha noción nos remite a una doble imagen de la organización, tanto a la que nos muestra su fragilidad como su estabilidad. La fragilidad, porque permite a los organismos estar listos para conjugarse en muchas posibilidades, en función de las sucesivas transformaciones a que da lugar el potencial de las uniones posibles y del uso de su libertad y grado de diferenciación por parte de los respectivos componentes. Su estabilidad, porque precisamente la anterior posibilidad de apertura mantiene vigente a la organización como tal.

Pero no es sólo su perfil, tal como lo hemos caracterizado, el distintivo de su peculiar funcionamiento. Además de las particularidades propias de cada contexto nacional, cada una de las instituciones de enseñanza superior se halla, a su vez, provista de sus propias características contextuales. Brennan y Shah (2000) identifican a las siguientes, como las más importantes fuentes de diferencia entre instituciones: su tamaño, su estructura organizativa, el prestigio, los recursos, la misión asignada, su historia y el tipo de liderazgo.

Sin embargo, aún podemos añadir otro elemento a la lista anterior, en relación a la cultura docente vigente en el común de dichas instituciones. El concepto de habilidades, de técnicas, de entrenamiento personal y desarrollo como docente se halla normalmente devaluado por un ambiente que prioriza la enseñanza teórica y las habilidades analíticas más que las habilidades aplicativas. Tradicionalmente, los docentes se han centrado en argumentar sobre el qué y no en el como. "El uso 
de técnicas concretas, habilidades, entrenamiento personal y desarrollo en la reestructuración de procesos era diversa, se reflejaba más en las experiencias individuales que en conocimientos compartidos sobre estos temas ni se reconocían estos aspectos como elementos a potenciar en el cambio organizacional", afirma Curri (2002).

De todo ello cabe deducir cuatro tipos de conclusiones provisionales. La primera, que las turbulencias sólo pueden traducirse en cambios efectivos si, además de ir acompañadas de un proceso político claro a nivel superior, se comprenden las dinámicas que los perfiles anteriores son susceptibles de introducir en todo proceso de mejora cualitativa. En segundo lugar, que dichos cambios, a pesar de aspirar a reunir los rasgos comunes de la transformación institucional, deberán ser alcanzados a partir de las propias dinámicas y procesos institucionales específicos de cada organización y contexto. La experiencia de una titulación, de una uni versidad o de un centro será un referente importante para los demás, pero no un modelo en el sentido convencional y aplicado del término.

En tercer lugar, que dichos rasgos reúnen características muy distintas, las cuales, de algún modo, van a ejercer su papel en el proceso emprendido: de organización (su funcionalidad y sentido), de naturaleza política (tipos de relación y de equilibrio entre poderes), de tipo psicosocial (liderazgo, motivaciones para el cambio, reconocimiento personal, autoimagen personal y colectiva), afectivas (filiaciones, grado de adhesión), cognitivas (comprensión de lo que se debe o quiere hacer), simbólicas (la trayectoria histórica reconocida, el valor de lo académico) y económicas (repercusiones personales en términos de recursos o prestigio, ganados o perdidos).

Finalmente, debemos concluir que afrontamos una realidad de cambio de naturaleza cualitativa centrada fundamentalmente en el concepto de formación y en la reconsideración de los procesos docentes, para desarrollarla de un modo funcional a las necesidades emergentes. Pero dicha formación debemos entenderla no sólo en el plano técnico, sino en un sentido mucho más profundo, en el de la necesidad de reelaborar la relación que se establece entre el conocimiento más relevante y el nuevo alumnado, en un sentido de desarrollo personal, en un sentido que potencie la dimensión cognitiva, la personal y creadora de los individuos.

Como vemos, de acuerdo con este diagnóstico, nos enfrentamos a un proceso cuyo rasgo más importante es el de ser de orden cultural, en vez de ser un proceso que pueda estar orientado sólo desde la racionalidad técnica. A su vez, esta reflexión nos puede ser de utilidad para comprender y evaluar el alcance del proceso de cambio en una institución de educación superior. Cuanto más sea un proceso técnico, más cerca se permanecerá del punto de partida, aunque la ilusión resultante sea la percepción de rapidez y de logro de los objetivos funcionales. Por el contrario, cuanto más se avance en la revisión de la actual cultura docente y en sus alternativas, más complejo se hará el proceso y el camino recorrido será mayor, aunque la ilusión resultante sea de una cierta crisis y de dificultad de obtener logros.

Ello supone alejarse de los modelos de gestión basados en la reestructuración organizativa y, por el contrario, adoptar modelos más complejos que combinen presión externa, sentido de proyecto, liderazgo con visión del mismo, participación y apoyo institucional formativo a los diversos agentes participantes en el proceso emprendido. 


\section{TEORÍAS QUE ILUMINAN LA NOCIÓN DE LOS PROCESOS DE CAMBIO EN LAS ORGANIZACIONES}

Veamos algunas de las teorías que permiten explicarnos cómo instituciones complejas evolucionan hacia el cambio. Dichas teorías, complementarias entre sí, nos permiten tener una perspectiva relativamente comprensible de unos procesos de cambio que, a priori, pueden parecer confusos y enormemente complejos.

\section{Teoría de los intereses de grupo}

Ésta es una corriente que en los USA se aplica fundamentalmente a corporaciones e instituciones. El punto de partida es que en organizaciones como las grandes corporaciones, en universidades, etc., se configuran grupos cohesivos para alcanzar favores y conseguir ventajas y de que dichos grupos poseen tanto dinámicas internas como constricciones provenientes del exterior. Baldridge propone un modelo en el que recoge los parámetros mediante los cuales se articulan los distintos intereses y permite comprender cómo éstos se transfieren al plano normativo explícito en el proceso de definición y de ejecución de las políticas organizacionales.

Se considera que cada uno de los aspectos tomados en cuenta puede realimentar al anterior y ser fuente potencial de nuevos conflictos, lo que nos Ileva a una representación de un circuito de naturaleza abierta, que se va modificando o realimentando progresivamente.

\section{Modelo de análisis político propuesto por Baldridge (1994:64)}

Dicho modelo enfatiza las relaciones de tipo político presentes en un contexto organizacional determinado.

Factores del contexto social

Articulación de intereses

Transformaciones normativas

Políticas

Ejecución de las políticas
¿Cuáles son las condiciones sociales que promueven la formación de valores divergentes y de grupos de interés?

¿De qué modo los distintos grupos de interés aguantan las presiones?

¿De qué modo las distintas presiones se traducen en una política oficial?

¿Cuál es el grado de compromiso explícito con ciertos objetivos y valores reconocidos?

¿Cómo se ejecuta el proceso de la política de la organización?

\section{Modelo de dependencia de los recursos}

En este modelo las organizaciones son percibidas principalmente como reactivas: si un cambio en el ambiente implica el surgimiento de relaciones críticas, la organi- 
zación deberá adaptarse hasta llegar a un mayor equilibrio que garantice el flujo de los recursos. Las organizaciones tienen una mayor capacidad de cambio pero su respuesta a las demandas del ambiente no es automático ni pasivo sino activo y de carácter volitivo (Heine, 1999).

La perspectiva de la "dependencia de los recursos" expone que una organización responde a las demandas externas debido a que:

- Las organizaciones acostumbran a estar en una posición de interdependencia.

- Las organizaciones no necesariamente dejan de ser maleables a las demandas externas.

- Las organizaciones tienen otras opciones aparte de conformarse de las demandas externas. Ellas pueden gestionar sus dependencias de muchas formas.

- Los ambientes no se tratan como realidades objetivas. El modo cómo una organización es definida, depende también de cómo esta es percibida y cómo es interpretada desde su contexto.

- Los contextos pueden realizar demandas no suficientemente relevantes.

- La mayoría de organizaciones se encuentran en contextos complejos confrontados a demandas competitivas y deben desarrollar diferentes criterios sobre cómo determinar el funcionamiento de la organización en relación a ellas.

La perspectiva de la dependencia de recursos muestra que para comprender las organizaciones se debe comprender el modo cómo éstas se relacionan con otros actores sociales desde su propio contexto. Se trata de un punto de vista político de la inter-intra interacción organizacional y esta teoría pone el énfasis en cómo las organizaciones actúan estratégicamente y escogen activamente su dependencia respecto de aquellas partes de su ambiente que consideran como recursos vitales.

En cualquier caso, de lo anterior se desprende que las respuestas organizativas hacia las presiones ambientales van a depender de las siguientes condiciones "externas" (Gornitzca, 1999):

- Cuál es la razón de que se ejerza una presión (la causa)

- Quién la ejerce (poder constituyente)

- En qué consiste dicha presión (contenido)

- De qué modo y a través de qué medios se ejerce (control)

- Dónde tiene lugar (contexto)

Todo ello implica que es necesario considerar y examinar el modo cómo las organizaciones perciben su ambiente, cómo actúan para controlar y evitar dependencias, cuál es el rol de las relaciones dentro de la organización en los diferentes procesos, y es de interés saber cómo la distribución de energías internas afecta y es afectada por las dependencias externas.

\section{Modelos culturales o antropológicos}

Desde otra concepción distinta, a partir del análisis de Ball (1989) y las aporta- 
ciones de Noble y Pym (1994) y Baldridge (1994), podemos considerar relevante atender esencialmente a la cultura de centro y analizarla desde dos dimensiones importantes. La primera sería la que relaciona el grado de autonomía de los agentes con el grado de control existente (eje de la autonomía-control). La segunda considerará el grado en que se comparten las prioridades en el seno de la misma, y las vinculan con el grado de consenso-conflicto existentes (eje consenso-conflicto).

Fig. 1: Espacios definidos por la cultura organizativa

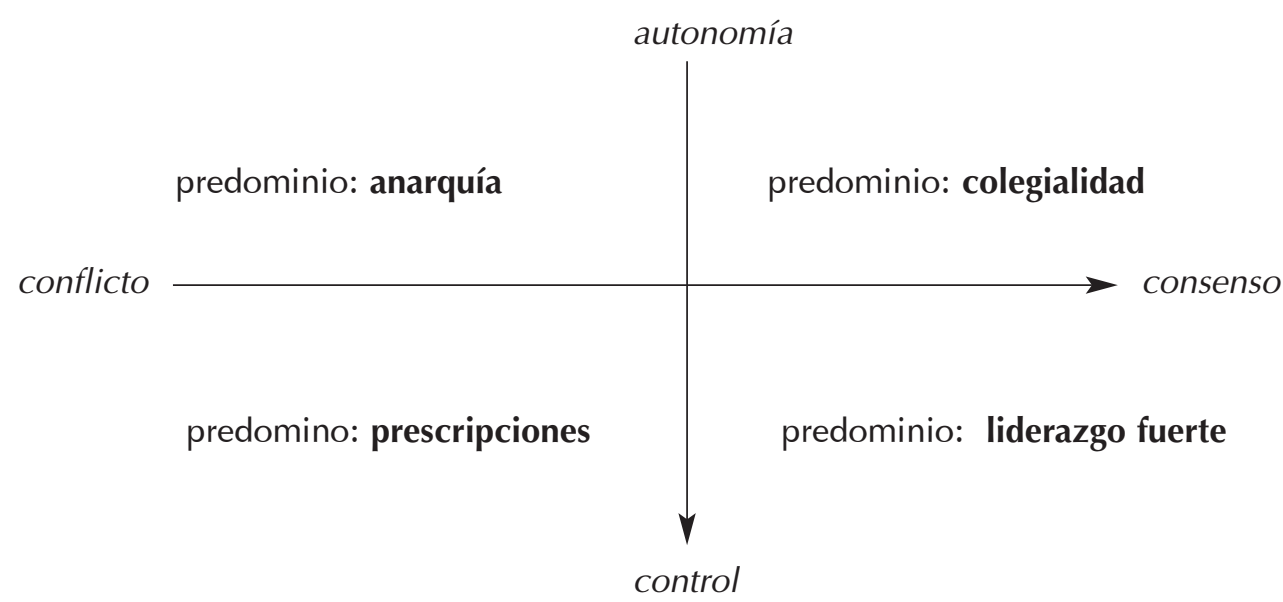

El cruce de ambos ejes y su fuerza da lugar a una tensión que se resuelve atendiendo a determinados rasgos predominantes en la organización, los cuales definen la cultura de cada centro, dando lugar a cuatro escenarios organizativos. Si existe autonomía y consenso, las relaciones serán de colegialidad; si en una situación predominan el control y el consenso es que hay un fuerte liderazgo; si se combinan un fuerte control con una situación de conflicto, seguramente la organización está dominada por las prescripciones; pero si dominan la autonomía y el conflicto, probablemente nos encontramos con una organización anárquica.

Los aspectos culturales de la organización influyen significativamente en los procesos de cambio organizacional. En coherencia con ello, y en la perspectiva de orientar dichos cambios, se deberá partir de una contextualización de los procesos y de los datos de ellos derivados, a partir de la realidad de cada cambio, de considerar y analizar su significado para los agentes implicados en ellos y adoptar una perspectiva de evolución histórica sobre un conjunto de referentes socialmente construidos.

El enfoque cultural de los cambios en las organizaciones de educación superior sitúa el foco de atención en los elementos cognoscitivos y normativos del ambiente que se forma en las acciones de la organización. En este sentido, dicha concepción nos permite comprender las resistencias al cambio como fenómenos conocidos en psicología social como "perseverancia de la creencia" (belief perseverance) la cual indica que no solo los esquemas básicos están en conflicto, sino que se deben atacar principalmente las creencias u opiniones acerca estos cambios. Al valorar las 
resistencias individuales al cambio deben valorarse también las que se relacionan con la cultura de los grupos de trabajo que se hallan en un nivel intermedio.

En una reciente investigación, Allen (2003) sugiere que es de una importancia fundamental que las instituciones de Educación Superior gestionen los procesos de cambio mediante el uso de técnicas que reflejen la compleja naturaleza de las instituciones universitarias; técnicas que reduzcan la inseguridad organizativa del trabajo y consideren a la organización conjuntamente como una comunidad. Entre los aspectos que inciden en esta percepción de inseguridad, el autor detecta los siguientes: su grado de predictibilidad, su grado de apertura, el grado de participación en los mismos, la naturaleza incremental o discontínua del cambio y si las decisiones se aplican mediante un poder coercitivo o persuasivo (p.84).

\section{5. ¿QUÉ HEMOS APRENDIDO DE OTRAS REFORMAS? ¿CÓMO AVANZAR?}

Una primera lección aprendida de otros procesos de reforma educativa es que los cambios educativos y organizacionales pueden ser una acomodación más o menos formal a la nueva situación que una realidad, es decir, un cambio que obedezca a una nueva lógica, que desarrolle un nuevo paradigma. Desde nuestro punto de vista, este es el aspecto central a evitar. Para ello, será necesario apuntar hacia unos procesos de elaboración que rehuyan cualquier tipo de tratamiento burocrático del problema, sin ir más al fondo de las cuestiones. Por ejemplo, para traducir los créditos actuales en ECTS, o créditos europeos, no basta con realizar una tabla de conversión del trabajo actual propuesto a los alumnos para ver su equivalencia en el formato ECTS. No basta, porque el modelo es otro: no es lo mismo un modelo centrado en enseñar que otro centrado en la acreditación del aprender y de lo ya sabido.

Deberemos reconocer que los modelos organizativos de docencia vigentes tienen su funcionalidad en el enfoque actual de las enseñanzas en Educación Superior, pero no van a perderla cuando el modelo de docencia y de aprendizaje se reoriente hacia las premisas ya apuntadas y basadas en una reorientación profesionalizadora, hacia el desarrollo de competencias y una reestructuración de las carreras. Ello supondría olvidar que los modelos actualmente vigentes se han desarrollado en función de las prestaciones del sistema tal como lo hemos conocido y de los requerimientos que se le hacían, y que son precisamente dichos requerimientos y prestaciones los que se propone que entren en transformación.

En consecuencia, en este nuevo proceso Europeo que se abre, el cual podría ser considerado como de "nivelación por arriba", se nos plantea la necesidad de reelaborar los aspectos curriculares y organizativos de la formación sin perder de vista que, a su vez, ambos aspectos dependen de algo fundamental, la capacidad de las universidades de enriquecer las condiciones de aprendizaje del alumnado, sea en el aula, sea en los diversos espacios tutelados o bien en su trabajo autónomo como alumnos. Con el añadido, además, de considerar este aprendizaje desde un enfoque orientado a priorizar un perfil de competencias a desarrollar en una formación dada.

En cualquier caso, las premisas que acabamos de apuntar nos indican la complejidad del camino a recorrer, lo cual convierte el modo cómo nos proponemos recorrerlo en un aspecto crucial del proceso. Para ello, nos serán de utilidad las orientaciones surgidas de experiencias precedentes de cambios curriculares y cuyo resumen de recomendaciones lo podríamos desglosar en dos grupos. El enfoque de naturaleza sociocultural, proveniente de investigaciones y de reflexiones como las 
de Fullan (1993, 2002) Hargreaves (1998, 2001), Curri (2002) y Bergquist (1992) nos apunta los siguientes referentes.

\section{ALGUNOS REFERENTES ESENCIALES PARA LOS PROCESOS DE CAMBIO EN LA ENSEÑANZA}

\begin{tabular}{|c|c|}
\hline Cambio & $\begin{array}{l}\text { Para cambios relativamente complejos, que supongan nuevos } \\
\text { modelos, nuevas pautas culturales para las acciones, los modelos } \\
\text { gerencialistas, top-down, no funcionan. Por el contrario, el cambio } \\
\text { es una resultante de una combinación entre arriba (los agentes que } \\
\text { lo lideran) y abajo (los agentes involucrados que lo realizan), y } \\
\text { un producto derivado de la acción de las presiones internas y las } \\
\text { externas. } \\
\text { Las personas no empiezan a desarrollar adaptaciones para el cam- } \\
\text { bio hasta que no se hallan envueltas en él (Fullan,1993). }\end{array}$ \\
\hline Conflicto & $\begin{array}{l}\text { En los cambios educacionales es necesario que los conflictos sean } \\
\text { asequibles (manejables) (M. Fullan 1993). } \\
\text { El mismo autor indica que el conflicto es esencial para el cambio. } \\
\text { Recuerda que los cambios educacionales implican nuevas habili- } \\
\text { dades, nuevas maneras de ser, nuevas creencias y nuevas com- } \\
\text { prensiones. } \\
\text { Los procesos de cambio comportan conflicto, (cognitivos, afecti- } \\
\text { vos, de saber hacer) que afectan a la autoestima personal y a las } \\
\text { relaciones sociales entre profesionales. }\end{array}$ \\
\hline Regulación & $\begin{array}{l}\text { Es fundamental atender a la regulación de los posibles conflictos } \\
\text { en individuos y grupos. }\end{array}$ \\
\hline $\begin{array}{l}\text { La } \\
\text { participación } \\
\text { y la } \\
\text { colaboración }\end{array}$ & $\begin{array}{l}\text { Reforzar la relación colaborativa entre agentes y compartir proce- } \\
\text { sos entre contextos equivalentes. Bergquist (1992) señala que la } \\
\text { "autonomía trae alienación y no colaboración". }\end{array}$ \\
\hline $\begin{array}{l}\text { Sobre la } \\
\text { organización } \\
\text { resultante }\end{array}$ & $\begin{array}{l}\text { Los datos de la investigación desarrollada por Curri (2002) expli- } \\
\text { can que existe una relación entre dirección, recursos humanos, } \\
\text { reestructuración, desarrollo organizacional, presiones externas } \\
\text { para el cambio y cambios organizacionales. Para llegar a un ver- } \\
\text { dadero cambio organizacional deben estar presentes más de un } \\
\text { factor y la congruencia entre distintos factores es un punto critico. } \\
\text { Las nuevas ideas provienen de los argumentos y los desacuerdos y } \\
\text { nos llevan al cambio. }\end{array}$ \\
\hline
\end{tabular}




\begin{tabular}{|l|l|}
\hline $\begin{array}{l}\text { Cambio } \\
\text { estratégico }\end{array}$ & $\begin{array}{l}\text { Es dependiente y centrado en la acción, mediante el aprendizaje } \\
\text { de una acción compartida en una dirección institucional } \\
\text { (Fullan 1993). } \\
\text { El concepto de mejora se va a contextuar en cada una de las dis- } \\
\text { tintas realidades organizativas. } \\
\text { No hay más calidad que la de la propia práctica de los profesionales. } \\
\text { Algunos cambios sólo se observan a partir de un plazo medio de } \\
\text { tiempo. }\end{array}$ \\
\hline \multirow{2}{*}{$\begin{array}{l}\text { Endividuos los cambios educacionales los individuos y los colectivos deben } \\
\text { tener el mismo estatus y poder. Fullan (1993) destaca que es impo- } \\
\text { tante permitir las discusiones de grupos para encontrar soluciones } \\
\text { y ejercitar su voz colectiva. }\end{array}$} \\
\hline
\end{tabular}

Una segunda línea de reflexión, de naturaleza más socio-constructivista, complementaria de las anteriores, y que ha tenido un impacto importante en los últimos años en la promoción del cambio en las instituciones educativas, ha sido la conocida como Investigación en la Acción. De esta línea de desarrollo profesional proponemos considerar algunos referentes que consideramos básicos a tener en consideración en los procesos de cambio, cualquiera que sea los que se emprendan:

- Profundizar entre los agentes involucrados en el proceso, un sentido de agencia, en su desarrollo como agentes políticos, es decir, con sentido de iniciativa pública.

- Desarrollar los procesos de cambio de acuerdo con los criterios de la poiesis aristotélica en vez de las pretendidas legitimaciones y eficacia derivadas de un enfoque basado en la thecné.

- Insistir en el carácter de razonamiento público de las deliberaciones y los argumentos, centrándose en las respuestas que tratan de profundizar en los principios de calidad del Sistema, de acuerdo con los principios del apartado anterior.

- Articular el diálogo en red, como forma fundamental de socialización para profundizar en la construcción de los acuerdos y en la calidad de los referentes, en el sentido cooperativo que propone Elliott (1990).

- Articular la reflexión desde la noción de proyecto compartido, como estructura de intenciones articuladas y explicitadas cuyo fin es incidir en la transformación de un contexto dado.

\section{EL PROCESO INICIADO EN LA UAB, SUS PRIMEROS PASOS}

Si tuviéramos que definir el modelo de trabajo adoptado en la UAB para iniciar el camino de la reforma de las titulaciones, éste se desarrollaría a partir de una adaptación del modelo sobre los espacios de la cultura organizativa, que hemos visto más arriba, y que describimos de acuerdo con el esquema siguiente: 
Esquema 1: Estrategias para desarrollar el consenso

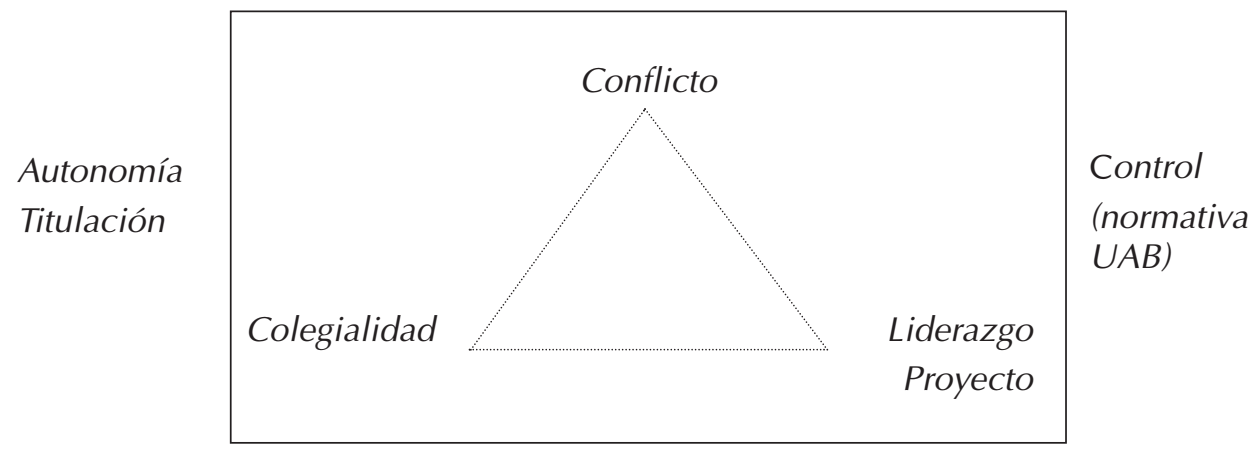

Apoyo técnico y logístico

institucional

La pretensión es llegar a propuestas de consenso amplio, tanto en el seno de cada titulación como con las autoridades responsables de los aspectos académicos. Ahora bien, para llegar a este consenso se requiere llegar a trabajar desde un punto en el que se respete la autonomía de los equipos, a la vez que dicha autonomía se somete a la regulación de la norma general de la $U A B$, descrita en términos de requisitos temporales, reconocimiento de responsabilidades, apoyo instrumental a la reflexión y de demanda de responsabilización y acomodación a unos criterios básicos de acreditación (Haug, 2003), según establece el modelo ECTS.

Para ello, se ha organizado el trabajo de reconversión de las titulaciones al nuevo modelo desde la perspectiva de desarrollar un proyecto específico $U A B$, lo cual ejerce un cierto liderazgo en el análisis y desarrollo de la propuesta. Dicho liderazgo se hace visible en dos tipos de actores, en la coordinación de la titulación que ejerce un liderazgo técnico y político, y en la comisión de titulación que le da apoyo, la cual va a ejercer también un liderazgo de tipo político sobre el resto de los componentes de la plantilla de profesores de la titulación.

Es necesario especificar que, al proponer la comisión interna de titulación como organismo de deliberación, representativo de las distintas sensibilidades presentes en cada una de ellas, nos hemos acercado, sin cumplirlas todas, a las propuestas de Cullinan y Hampton ${ }^{5}$, los cuales recomiendan los siguientes rasgos deseables en un equipo de trabajo:

- Incorporar a personas productivas, a quienes les afecten los cambios.

- Incluyan a un escéptico en el equipo.

- Establezcan un balance adecuado en el seno del equipo entre las diversas sensibilidades para obtener una amplia representación de los diversos puntos de vista.

5. Citado por Engelkemayer, en Susan W. y Elaine Landry (2001) 
- Escojan a miembros del equipo que se perciban como creíbles por los demás en su institución.

- Escoja a miembros del equipo que posean las habilidades necesarias para negociar con los demás, sean del propio departamento o de otros.

- Si es posible, incluya a un alumno en el equipo.

No obstante lo anterior, el proceso que se inicia se halla todavía en sus primeros momentos puesto que ha empezado en el pasado mes de octubre, por lo que tenemos pocas concreciones a aportar, aunque una primera valoración de urgencia sobre los primeros pasos caminados nos mantiene en la validez del modelo. Dicho modelo lo podemos ver especificado a través de los aspectos siguientes.

- Finalidad: Elaborar un modelo de proceso de transformación de las actuales titulaciones en titulaciones-ECTS en el plazo de tres años, hasta el 2006.

- Responsabilidad política: Vicerrectorado de Asuntos Académicos.

- Responsabilidad técnica: orientar y dar apoyo técnico al proceso. Unidad de Innovación docente en Educación Superior (IDES), recientemente creada por el Consejo de Gobierno de la universidad. Los responsables de esta Unidad establecen, a su vez, una coordinación entre titulaciones y entre el proceso y el Vicerrectorado.

- Sensibilización, divulgación: desarrollar una línea general de información sobre los ECTS para toda la universidad, mediante conferencias de expertos, documentos en red, divulgación en folletos, etc., y complementado todo ello con acciones formativas voluntarias más específicas para coordinadores de titulaciones distintas a las de la fase piloto. También se crea una web para difundir la documentación más relevante que facilite esta sensibilización entre todo el profesorado.

- Marco de referencia para la actuación: elaboración de un documento estratégico general centrado en una introducción explicativa y la previsión de tres momentos para el desarrollo de las propuestas curriculares: un momento macro, en el que las titulaciones negocian y establecen las líneas generales de competencias a desarrollar en su alumnado, un nivel meso, en el cual las líneas anteriores se concretan en competencias para las materias y se especifican las líneas generales de la acción didáctica y un nivel micro en el cual se va a entrar en el detalle del tiempo y en el de la acreditación del trabajo del alumno, para, a su vez, calcular el tiempo del profesor. Dicho documento marco, se va elaborando a partir de un esquema inicial consensuado y a partir de las dinámicas que se van registrando en el proceso de pilotaje de las titulaciones seleccionadas para iniciar el proceso.

- Objetivo: asumir y desarrollar una propuesta de plan docente para cada titulación adherida voluntariamente al proceso, que se halle de acuerdo con los requisitos-marco UAB para la transformación de una titulación de acuerdo con los créditos ECTS.

- Proceso inicial: selección de cuatro titulaciones-caso, para derivar de ellas el conocimiento práctico de todo el proceso de concreción de esta transformación y que pueda ser de utilidad para aplicar en la segunda oleada de titulaciones transformadas en ECTS. 
- Fase de pilotaje:

- Seguimiento de los procesos de reflexión surgidos en estas titulaciones para incorporarlas al documento general, cuando se consideren pertinentes y relevantes.

- Apoyar a los coordinadores de titulación en sus necesidades, con recursos instrumentales y apoyo en la deliberación respecto a los caminos a seguir, cuando lo necesitan.

- Elaborar propuestas de progreso para las titulaciones, cuando lo requieran, y dentro.

- Estimular a las titulaciones a mantenerse dentro de los parámetros anteriores.

- Acciones complementarias: paralelamente al proceso anterior, la Unidad IDES desarrolla líneas de acción, formativas sobre estrategias didácticas, @learning, elaboración de materiales, etc., todo ello con el concurso de docentes y grupos que han adoptado este marco como referente para su propia acción profesional.

\section{BIBLIOGRAFÍA}

ALLEN, D.K. (2003). Organisational climate and strategic change in Higher Education: Organisational Insecurity. Higher Education, 46, 61-92.

BALDRIDGE, J.V. (1994). Building a political model. En BUSH, T. (ed), Managing education. Theory and practice, 57-66. Buckingham: Open University Press.

BALL, S.J. (1989). La micropolítica de la escuela. Barcelona: Piados

BERQUIST, W.H. (1992). The four cultures of the academy. Insights and strategies for improving leadership in collegiate organizations. Barcelona: Jossey-Bass.

BOURDIEU, P. (1997). Razones prácticas. Barcelona: Anagrama.

BRENNAN, J. y SHAH, T. (2000). Changing faculty roles in research university. Using the pathways strategy. Change, 32 (6), 42-45.

CASTELLS, M. (1999). La era de la información, economía, sociedad y cultura. Madrid: S. XXI.

CASTELLS, M. (2002). La galaxia internet. Barcelona: Plaza Janés.

CURRI, GUNDRUN (2002). Reality versus perception: Restructuring tertiary educational and institutional organisational chage. A case study. Higher Education, 44, 133-151.

ELLIOTT, J. (1990). La investigación acción en educación. Madrid: Morata.

ENGELKEMEYER, S.W. y LANDRY, E. (2001). Negotiating Change on Campus. AAHE Butlletin, 53 (6).

EUROPEAN COMISSION (2003). Communication from the Comission: "Education \& Training 2010" the success of the Lisbon strategy hinges on urgent reforms. Brussels: Comisión Europea, 11/11/2003 COM (2003) 685.

FULLAN, M. (1993). The complexity of the change process. En FULLAN, M. (ed), Change Forces. Probing Depths of Educational Reform, 19-41. Bristol: Falmer Press. 
FULLAN, M. (ed) (1993). Change Forces: Probing Depths of Educational Reform. Bristol: Falmer Press.

FULLAN, M. (2002a). Liderar en una cultura de cambio. Barcelona: Octaedro.

FULLAN, M. (2002b). Los nuevos significados del cambio en educación. Barcelona: Octaedro.

GRANT, K. (2003). Making sense of education change at Thirstle College. The existence of witchcraft, witches and shamans. International Journal of educational Management, 17 (2), 71-83.

GORNITZKA, A. y MAASSEN, P.A.M. (eds.) (1999). Interim Report to the Euopean Commission. DG. XII. Enschede: Center for Higher Education Policy Studies (CHEPS).

GUMPORT, P.J. (2000). Academic restructuring. Organizational change and institutional imperatives. Higher Education, 39 (1), 67-91.

HARGREAVES, A. (2001). Aprender a cambiar. La enseñanza más allá de las materias y los niveles. Barcelona: Octaedro.

HARGREAVES, A. (ed) (1998). International handbook of educational change. Dordrecht: Kleuwer Academic.

HAUG, G. (2003a). Quality Assurance/Accreditation in the emerging European Higher Education Area. A possible scenario for the future. European Journal of Education, 31 (3), 229-240.

HAUG, G. (2003b). Conferencia UAB, 10/11/03.

HEINE (1999). Theoretical framework. En GORNITZKA, A. y MAASSEN, P.A.M. (eds.). Interim Report to the Euopean Commission. DG. XII. Enschede: Center for Higher Education Policy Studies (CHEPS).

JELIAZKOVA, M. y WESTERHEIJDEN, D.F. (2002). Systemic adaptation to a changing environment. Towards a next generation of quality assurance models. Higher Education, 44 (3-4), 433-448.

NOBLE, T. y PYM, B. (1994). Collegial Authority and the receding locus of power. En Bush, T., (ed), Managing education. Theory and Practice, 28-43. Buckingham: Open University Press.

RUÉ, J. (2003). Action Research in Education, in the Era of Liquid Modernity. Educational Action Research Journal, 11 (2), 197-212.

WEICK, K.E. (1994). Educational organisations as loosely coupled systems. En Bush, T. (ed), Managing education. Theory and Practice, 118-131. Buckingham: Open University Press. 